\title{
PSICOTERAPIA PSICOANALÍTICA ACTIVA
}

\author{
Jaime D. Llancán Obispo*
}

\begin{abstract}
Resumen
Es una técnica que parte de las observaciones realizadas al trabajo de Freud, desde Ferenczi, Sarkissoff hasta Llancán; se basa fundamentalmente en la actuación tanto del terapeuta como del paciente. La actuación considera dos aspectos: la relación transferencial y la actuación propiamente dicha de la relación entre ambos. El terapeuta no permanece pasivo en la escucha de su paciente, la terapia se realiza en una relación corporal frente a frente; en muchos momentos el paciente necesita ser apoyado corporalmente, para ello el terapeuta lo sostiene y apoya con su cuerpo, lo acompaña con la voz cuando el paciente necesita y desea gritar, llorar, golpear, cuando necesita un abrazo con amor, etc. El terapeuta facilita la actuación de su paciente en la externalización de sus contenidos tanto conscientes como inconscientes. La técnica considera la energía corporal, el vínculo amoroso desde la infancia, el manejo de los afectos en el presente, la empatía, el contacto físico, el insight, la resistencia, el mental.
\end{abstract}

Palabras clave: contacto físico, vínculo amoroso, externalización, actuación.

\begin{abstract}
It is a technique that takes the observations to Freud's work, from Ferenczi, Sarkissoff until Llancán. It is mainly based on both the therapist and the patient conduct. This conduct considers two aspects: the transference relation and the conduct itself, of the relation between both of them. The therapist does not remain passive while listening to his patient; the therapy is done in a corporal relation face to face. In many moments, the patient needs to be supported corporally. In order to do it, the therapist holds him and supports him with his body, accompanies him with the voice when the patients needs and wants to scream, cry, hit, when he needs a hug with love, etc. The therapist facilitates his patient conduct in the externalization of his inner feelings both conscious and unconscious. The technique considers body energy, the love bond since childhood, the handling of affection in the present time, empathy, physical contact, insight, resistance and the mind.
\end{abstract}

Key words: physical contact, love bond, externalization, conducts.

\footnotetext{
* Psicólogo Psicoterapeuta. Psicoanalista. jaimellancan@hotmail.com
} 
Al hablar de la psicoterapia psicoanalítica activa, estamos hablando de la actuación tanto del paciente como del terapeuta, dentro de la situación y el proceso terapéutico.

Freud (1905/1981) descubrió el fenómeno de la actuación en relación a la transferencia en Dora y lo describió en "Fragmentos de análisis de un caso de histeria (1905)". Después de su trabajo "Recordar, Repetir y Elaborar" (1914) habla de la actuación como derivada de la situación terapéutica y de la transferencia, "El paciente no recuerda nada nuevo de lo olvidado y reprimido, sino que lo actúa, no lo reproduce como un recuerdo, sino como una acción, lo repite sin saber desde luego que lo hace" (Freud, 1914/1981, p. 1684).

Freud siempre trabajó sobre este entendimiento de la actuación, es por ello que pensó que el propósito de estar en el diván era bloquear el campo expresivo motor de la vivencia y de la conducta: por la limitación del movimiento quería interrumpir la descarga hacia afuera e incrementar la presión hacia adentro con el objeto de facilitar el recordar. Esta técnica fue llevándolo a Freud a una creciente pasividad terapéutica, por la intelectualización, el psicoanálisis se estaba convirtiendo en una disciplina académica y pedagógica, la relación con el paciente carecía de todo contacto afectivo y físico. La neutralidad terapéutica, que es la piedra angular en la psicoterapia psicoanalítica, fue creando cada vez más distancia afectiva entre terapeuta y paciente, ya que la pasividad e inactividad terapéutica eran justificadas por aquellos que pedían objetividad y neutralidad científica en el psicoanálisis.

Coderch (1987) analiza la relación entre transferencia y actuación, la transferencia en la repetición de una serie de comportamientos de parte del paciente en la persona del terapeuta, de las relaciones que el paciente tuvo en su infancia con sus personajes significativos. Por lo tanto no cabe la menor duda de que la actuación es un fragmento de la transferencia, aún cuando no toda la transferencia haya de ser considerada como pura actuación. Consideraque la actuación es la puesta en acción de los conflictos inconscientes por parte del paciente, en lugar de vivirlos en la transferencia.
Como se mencionó en el primer párrafo, la psicoterapia psicoanalítica activa tiene una relación directa con la actuación.

La actuación es estudiada y entendida en dos posiciones diferentes que se contraponen: para algunos el actuar en terapia psicoanalítica tiene una valoración negativa, poniendo en claro que es más fácil trabajar con palabras que con hechos; así, el actuar es considerado como una expresión del comportamiento indeseado y puede poner en peligro la psicoterapia psicoanalítica, esto ha favorecido que el actuar tenga una connotación negativa.

A pesar de su consideración negativa, el terapeuta influye en el surgimiento del actuar a través del encuadre, las normas establecidas, la regla fundamental, y, el trabajo con la palabra a través del recordar. Es la segunda posición.

Ferenczi (1925) observó con mucha preocupación la lentificación y el tiempo largo que demoraba la terapia psicoanalítica practicada por Freud. Creyó que al poner primordial importancia la experiencia emocional en la terapia se lograría tener terapias de corto tiempo. Propuso incrementar el contenido emocional en las sesiones, e intensificarlo con mayor frecuencia, desafiando la creencia de que el terapeuta debía ser una pantalla en blanco, lo cual levantó muchos cuestionamientos, específicamente de Freud.

Ferenczi (1925) recomendó que el terapeuta debe involucrarse activamente con los pacientes durante todo el tiempo que dure la terapia, también opinaba que los pacientes vulnerables necesitaban menos de interpretaciones e integridad científica y más apoyo, aliento y nutrición terapéutica. Sostenía sus ideas sobre la teoría, que las carencias y conflictos de la niñez llevaban a la neurosis. Como consecuencia, él creía que los pacientes profundamente afligidos o desequilibrados a veces necesitaban ser abrazados y reconfortados físicamente y que negarles esto sería cruel. Ferenczi sugirió que la actividad terapéutica podría enmendar las fallas parentales tempranas que el paciente había vivido y que había llevado a la terapia. Asimismo recomendó intervenciones activas y flexibles; creyó que Freud se estaba volviendo pedagogo, al enseñar a sus pacientes lo que sus síntomas significaban y representaban al paciente. Ferenczi estableció una nueva relación terapéutica que 
es cooperativa, así le daba más poder a sus pacientes y la relación era de igualdad, en el sentido que ambos participaban activamente, propuso además que todos los pacientes que pedían ayuda debían recibirla, y que dependía del terapeuta decidir e idear el mejor curso de acción terapéutica.

Ferenczi fue un crítico de la técnica Freudiana, trazó un paralelo entre el niño traumatizado por la hipocresía de los adultos, la persona mentalmente enferma por la hipocresía de la sociedad y el paciente, cuyo trauma es revivido por la hipocresía profesional y la rigidez técnica del terapeuta.

Boesky (1982) refiere: "Es imposible expresar en palabras todos los aspectos de la experiencia" (p. 210).

Laplanche y Pontalis (1972) refieren que la actuación tiene dos significados:

a.- La actuación en la transferencia.

b.- El recurso a la acción motora.

Esta combinación se relaciona con el modelo de los procesos cognitivos- afectivos en su relación con la motilidad. La actuación no sólo se relaciona con el recordar y el repetir.

Laplanche y Pontalis (1972) recomendaron una reconsideración en el psicoanálisis de la acción y de la comunicación que debería incluir los siguientes tópicos: la abreacción y control de afectos, e impulsos ciegos, vivir la vida, acción intencional, descarga motora, acciones altamente organizadas tales como el juego y la representación escénica, la estructuración de relaciones, los esfuerzos creativos y otras maneras de resolver tensiones y conflictos por medio de movimientos y acciones; la actuación como resultado y solución de posibilidades de defensa y adaptación en el repertorio de un individuo en relación con su ambiente.

Los procesos mentales ocurren en el aparato psíquico, desde el extremo de la percepción hacia el de la motilidad. Las expresiones afectivas y no verbales pueden calificarse como actuación.

Laplanche y Pontalis (1972) afirman: Freud describe la trasferencia al terapeuta como una modalidad de actuación. No encuentra la relación entre fenómeno de repetición en la transferencia y las manifestaciones de actuación.

El paciente actúa ante nosotros en lugar de informarnos, la actuación también ocurre fuera de la transferencia, cuando el paciente se entrega a la compulsión a repetir, sustituye el impulso de recordar, no sólo en su relación con el terapeuta sino también en todas las actividades de su vida cotidiana.

La actuación surge como defensa y/o adaptación en el sujeto en relación a su medio. Hay condiciones inconscientes que pueden intensificar la tendencia a actuar como traumas tempranos, trastornos del sentido de la realidad, sensibilización visual, fijación en el nivel de la acción, estos ponen énfasis en el lenguaje de la acción, en contraste con el lenguaje verbal.

La acción puede producir un sentimiento de modificación de sí mismo más intenso e inmediato que las palabras, mayor es la posibilidad de influencia de la realidad externa. El actuar puede restituir el sentimiento de realidad. El actuar puede estar al servicio de la defensa, frente a deseos de pasividad y angustia, como también al servicio de la anulación de evidencias de impotencia y desamparo traumático.

P. Blos (1963) describe la actuación como una solución común y apropiada para los problemas de separación en el adolescente. También sostienen que la actuación está al servicio de la restauración del sentido de realidad.

Boesky (1982) habla de la actuación dentro del contexto de repetir y reelaborar, es decir el paciente repite en la situación psicoterapéutica para que el terapeuta observe sus comportamientos y lo ayude a reelaborar, este actuar de repetir es inconsciente, por lo tanto el comportamiento le trasmite el metamensaje: "ayúdame a superar esta experiencia".

Freud (1916/1981) en su artículo "Conferencia de introducción al psicoanálisis" sostuvo que la palabra fue el distintivo del tratamiento, la razón del diván era bloquear el campo expresivo motor de la vivencia y de la conducta, incrementando la presión hacia adentro para facilitar el recordar. La abstinencia y la frustración aumentan la presión interna y reavivan recuerdos retroactivos. 
Blum (1976) refiere que hay vivencias preverbales que no pueden expresarse por medio de la palabra. La palabra no es suficiente para expresar todos los aspectos de la experiencia, la actuación es el potencial para actualizar al paciente.

Rosenfeld (1965) sostiene que no puede haber terapia sin un cierto grado de actuación. Cuando se establecen reglas que contrarrestan la actuación, éstas pueden tener un efecto contrario y conducir dentro o fuera de la situación analítica conductas sustitutivas motivadas inconscientemente, difíciles de controlar.

Balint (1934) refiere que la actuación es el nuevo comienzo para acercarnos al cambio, mientras que la valoración negativa de la actuación se asocia con la repetición.

Eissler (1958, citado por Coderch, 1987) refiere que es imprescindible adoptar cualquier modificación que esté al servicio del cambio estructural, para ello es necesaria la actuación.

La actuación dentro o fuera de la situación terapéutica busca, con todos sus recursos, reconocimiento afectivo-corporal. La conducta actuada con sus rasgos impulsivos, complejos, corporales y regresivos, mayormente su motivaciones inconscientes, colocan al terapeuta frente a exigencias considerables. Poniendo buena atención a la dinámica actual y a los afectos y vivencias más cercanos, podemos reconocer mejor el pasado y a la vez a ser el presente más presente, es decir más libre del pasado.

Coderch (1987) refiere que cada paciente tiene un estilo propio de comunicarse, tiene una manera muy peculiar de expresar sus sentimientos, actitudes, estados de ánimo, conflictos, a través de mímica, gestos y su comportamiento en general dentro de la sesión. El terapeuta tiene que tener la habilidad de captar el mensaje que su paciente le trasmite a través de este tipo de comunicación no verbal: por la forma particular de expresarse como el tono de voz, el timbre, el arreglo personal, los ademanes, etc. todo este tipo de comunicación es acompañado por la palabra.

Asimismo, Coderch (1987) considera que hay dos tipos de comportamientos manifestados por el paciente, uno fuera de la sesión llamado el acting out y el otro dentro de la sesión llamado el acting in. La actuación es considerada por él como una descarga de deseos infantiles reprimidos fuera de la terapia. El acting out fue en su origen un concepto netamente psicoanalítico, con el correr del tiempo se ha considerado en el campo de la psiquiatría y de la psicología, refiriéndose a determinado tipo de comportamiento que manifiesta el paciente fuera de la terapia. El paciente actúa libremente de manera inconsciente, sin darse cuenta qué es lo que está repitiendo y nada tiene que ver en la relación con su terapeuta. De ahí que estos tipos de comportamientos inicialmente no son entendidos por el paciente que es una repetición de sus conflictos inconscientes.

El acting in es el actuar dentro de la sesión, tiene la ventaja de ser observado directamente por el terapeuta, estos comportamientos son parte de la transferencia, lo que facilita al terapeuta su análisis en lo que respecta a la relación transferencia a través de la interpretación, ya que el paciente es consciente de la manera cómo actúa frente a su terapeuta; sus acciones las justifica argumentando en relación a lo que considera de acuerdo a lo que él observa en relación a su terapeuta, e igualmente, como en el caso anterior, él no tiene la posibilidad de recordar sus conflictos inconscientes. Mayormente los conflictos inconscientes lo llevan a actuar de una manera impulsiva, generalmente destructiva para el propio paciente, siendo rechazado este tipo de comportamiento en el entorno familiar, laboral o social.

En estos dos tipos de comportamiento, la actuación se caracteriza por su carácter irracional y poco ajustado a la realidad y a las necesidades del propio paciente, una de las tareas del terapeuta es prevenir el surgimiento de estas manifestaciones comportamentales, trabajando adecuadamente tanto terapeuta como paciente.

La actuación del paciente tiene un doble significado, por un lado es una resistencia al descubrimiento del inconsciente, por otro lado es una fuente importantísima de información y una manera de comunicar al terapeuta cuáles son los conflictos que existen en el interior y cuáles las defensas con la que se intenta mantenerlos ocultos. En resumen la actuación puede tener un sentido predominantemente comunicativo, o bien una intencionalidad fundamentalmente destructiva de la posibilidad de diálogo y comprensión. 
Cuando una persona se encuentra en terapia, a través de la actuación ensaya cómo resolver la situación transferencial desplazándola al área del comportamiento, especialmente fuera de la situación terapéutica, buscando en ella nuevos caminos, ésta es la actuación que Coderch (1987) llama creativa. La actuación destructiva en cambio, es aquella que se opone frontalmente a la comprensión de la transferencia y se limita a reproducir ciegamente los conflictos con los personajes significativos de la primera infancia proyectados en el terapeuta.

El autor del presente artículo considera, cuando estudia la actuación y su relación con la transferencia, que el sujeto actúa de manera irracional, exteriorizando sus conflictos internos que pueden ser destructivos o perjudiciales para él. Todo ello es muy importante. Considera también que en todo el actuar del paciente hay que enfocarlo, no ponerle la lupa sólo al inconsciente; durante el proceso psicoterapéutico también actúa de manera saludable, evidenciando comportamientos racionales y adaptados, muchas veces relata su actuar en otras circunstancias, en donde sus comportamientos son favorables o saludables. Ello es muy importante, porque puede ser el punto de partida en el entendimiento de su propia problemática y darse cuenta de que su vida no todo es un drama, lleno de preocupaciones, conflictos y angustias; también ha vivido escenas donde actuó y ejercitó comportamientos saludables; sobre esta base el paciente tiene que practicar y actuar de manera similar a estos comportamientos, cuando está frente a sus conflictos.

La tarea consiste en que primero tiene que ser consciente de sus conflictos, de sus comportamientos transferenciales, para luego sobre el conocimiento de ello, actúe de manera saludable.

\section{DEFINICIÓN DE PSICOANÁLISIS ACTIVO}

Es un método activo donde el terapeuta, sin inducir, está dispuesto para acoger, interpretar y acompañar toda reacción, todo proceso corporal, emocional, verbal e infraverbal del paciente, analizando constantemente la relación transferencial, contratransferencial y las manifestaciones del inconsciente. Los logros alcanzados en el psicoanálisis tradicional se conservan en el psicoanálisis activo.

\section{SURGIMIENTO DEL PSICOANÁLISIS ACTIVO}

En 1919 Freud vio que los trastornos de los pacientes no sólo dependían de la represión, sino también de las múltiples circunstancias exteriores. Sobre estas reflexiones Sandor Ferenczi (1925) propone una metodología en donde el cuerpo deviene objeto y lugar de análisis, cada interpretación afecta la postura corporal del sujeto.

Después W. Reich (1940) elabora una teoría original y singular, donde el cuerpo deja de ser un espacio fantaseado, para convertirse en mayor medida en cuerpo real; poneénfasis a los contenidos sensoriales y sensitivos que se desprenden del cuerpo biológico y de su relación con el inconsciente. La represión provoca una disociación de las pulsiones, de los cuales una parte se dirige al propio yo formándose así la coraza muscular y psicológica que dificultan las explicaciones afectivas, emocionales (el cuerpo no puede expresarse adecuadamente a nivel verbal y no verbal).

Serge Peyrot (1988) creó la Terapia Mofoanalítica, es una terapia psico-corporal analítica, en la cual el terapeuta trabaja en cada instante con la unidad cuerpopsique de su paciente como un sistema integrado, donde el cuerpo psíquico, las sensaciones, las emociones, la espiritualidad, la expresión corporal-física, energética y verbal, constituyen una sola y única realidad del ser.

Finalmente Jean Sarkissof (1989) trabajó junto con Peyrot, estudió en profundidad la relación madre-hijo; considera los descubrimientos de Alice Miller (1983) sobre la educación represiva o pedagogía negra, la cual crea un traumatismo en el niño. Sarkissof estima que lo más grave de toda relación es la ausencia de empatía entre la madre y el hijo. Integrando los numerosos descubrimientos elabora así un psicoanálisis abierto, activo y dinámico, basado en la empatía, el amor, el sostenimiento, aceptando y comprendiendo tal como es el paciente.

\section{COMUNICACIÓN MADRE-HIJO}

La naturaleza ha privilegiado a la mujer, a la madre, quien cumple una de las grandes funciones primordiales en la existencia de todos los seres humanos: la de iniciar en su hijo la tarea fundamental de la comunicación, esta primera comunicación no es con el lenguaje hablado, es ante todo una comunicación corporal, hechos de impulsos, de júbilo, de 
intercambios empáticos, de emociones y sentimientos; haciéndola particularmente receptiva a todas las vibraciones afectivas, al intercambio de caricias y de contactos físicos. Introduce al niño en el universo de la comunicación preverbal, primordialmente corporal, con un lenguaje intuitivo cargado de afecto, que le ayuda a crear las bases del lenguaje hablado.

La presencia del padre aparece posteriormente, cuya función principal es la de introducir al niño en el universo del lenguaje, la palabra, el verbo.

El amor que le da la madre a su hijo es importantísimo, es el sello eterno con la cual va a convivir el niño. La mujer es diferente del hombre en su cuerpo, como también en el espíritu, en los sentimientos, en su manera de percibir, de sentir, de reaccionar, ante las diferentes circunstancias de la vida. La mujer es más intuitiva, menos sumisa a lo mental, está predestinada a entender a su hijo. No es casual que resulte tan sensible a las caricias, que son también las primeras palabras de amor de la madre a su hijo.

La afectividad de la madre es la más rica, la más profunda, la más vulnerable, la más significativa, la más poderosa, durante los primeros años de vida del niño; la madre percibe todas las sutilezas de ello. Para ella es un juego entrar con el niño, en el diálogo corporal, intuitivo y preverbal. Cada deseo, cada emoción, cada sentimiento, cada pensamiento del niño llegan a su madre; el niño recibe una respuesta y responde a su madre con nuevos estímulos. Asíla díada madre-hijo se baña dentro de un mundo de intercomunicación, de reciprocidad, de amor fusional, en donde se estructura el niño.

Una madre buena y saludable, tiene por naturaleza un elemento afectivo muy significativo, es la empatía, este elemento afectivo, es absolutamente indispensable para el desarrollo mental del niño. La naturaleza le ha dado al niño una extrema sensibilidad a la empatía primordial de la madre, lo que configura a ambos en esta relación diádica, en la relación perfecta madrehijo. Por ella existe, por ella se construye.

Desde el momento de la concepción, la madre empática comunica a su hijo el mensaje no verbal: «soy feliz de que estés aquí». El campo de comunicación de la madre armoniza la energía del niño, y va elaborando la imagen que tendrá más tarde de sí mismo. Su orgullo de existir es el reflejo del amor de su madre por él. Pero la madre desprovista de empatía y presencia destroza a su hijo. Los mensajes no verbales también, lo condena: «no comprendo nada de tu ser», el niño también es hipersensible a la ausencia de empatía materna, que es mortal para él.

Dependiendo de cómo haya sido la concepción deseada o rechazada, el niño lleva en secreto la convicción de su valor eminente o de su indignidad. El niño se siente feliz, ama a su madre, se siente bueno, se quiere a sí mismo, espera amor de los demás, siente que es importante para sí mismo y para los demás; es el caso del niño cuya concepción fue deseada. Por el contrario el niño siente que no se quiere, nadie lo quiere, sólo resaltan en él lo negativo, se toma por un desgraciado, indigno para los demás y para sí mismo; es el caso del niño cuya concepción no fue deseada.

La comunicación entre la madre y el hijo será por medio de la energía, esta energía es la fuente de la psique y del funcionamiento del cuerpo. La energía es armoniosa por naturaleza. Cuando la comunicación no es aceptada y comprendida por la madre, la energía topa con barreras mentales que traban y perturban su flujo, que entonces se transforman en caótico. El niño se siente ridículo, avergonzado o incluso culpable: sentimientos que afean y desfiguran la imagen de sí mismo. La vergüenza de sí es la manifestación de una energía negada, y trabada en su libre funcionamiento. Cuando los recuerdos de la infancia están cargados de fuertes emociones, se graban en el niño y permanecen en el inconsciente. Según Janov (1970), estas emociones, que nos traumatizaron y que hemos reprimido o cortado, quedan presentes en nuestro inconsciente, y pueden salir de allí con fuerza, haciendo revivir al paciente antiguas circunstancias traumáticas. Estas emociones, densas, y acompañadas de toma de conciencia, son los primales (la emoción primal, cuando se revive es menos dramática).

La madre buena y saludable es aquella que deposita en el hijo, el germen del optimismo en su interior, no las semillas de la desesperación. Cuando el niño siente que es amado, el amor nace en él, por sintonía de vibraciones. El ejercicio del amor y la comunicación son los antídotos de todos los males en el niño. 


\section{EL APARATO PSÍQUICO EN EL PSICOANÁLISIS ACTIVO}

El aparato psíquico, desde una óptica del psicoanálisis activo, es la misma del psicoanálisis tradicional, considerando la primera tópica: consciente, pre-consciente, e inconsciente; la segunda tópica: superyó, yo y ello; además considera dos elementos adicionales que funcionan dentro del aparato psíquico; el Mental que se conceptúa como la modalidad lineal deconcienciaque busca comprender la realidad a través de la mente racional e intelectual; también es definido como el aparato psíquico que graba todos nuestros recuerdos, posiblemente todas nuestras percepciones, desde el nacimiento hasta la muerte. Asimismo, es considerado como un contenido amenazante, peligroso, el cual convive entre nosotros, porque se encuentra en el inconsciente, es estimado como nuestro enemigo. El elemento mental crea nuestros errores, nuestras falsas ilusiones, nuestras mentiras, nuestras neurosis, nuestros miedos y también nuestros odios. El otro concepto, es el Primal, término desarrollado por Janov (1970), quien lo explica de la siguiente manera: cuando una experiencia del pasado, sea de persecución, sea depresiva ha sido demasiado dolorosa para ser elaborada, es reprimida y se queda dentro de nosotros, inconsciente, detrás de un corte protector. Cuando el análisis es suficientemente profundo, el corte se deshace, entonces toda la energía dolorosa reprimida reaparece en la conciencia, para ser elaborada esta vez.

Esta experiencia, muchas veces muy intensa, que resurge como en el pasado, acompañada de todas las percepciones de entonces se llama primal.

El elemento que dinamiza el aparato psíquico es la energía, sólo se la considera como tal.

Respecto a la patología, Bleger (1981) ha mostrado queen el fondo de cada paciente se encuentra un núcleo fetal, psicótico, que tiene la llave, no sólo de la psicosis, sino también según él, de la perversión, del masoquismo, de la psicopatía, de las enfermedades psicosomáticas y de las neurosis. Cuando se va a la profundidad de lo no verbal, este núcleo se deja alcanzar cuando se analizan las innumerables manifestaciones afectivas transferenciales.

La causa principal de todos nuestros trastornos y enfermedades, puede ser vista como una perturbación de una energía que, habiendo dejado de estar en armonía vibratoria con la totalidad de las energías, se convierte en caótica. La madre es la primera encargada de mantener o conducir el estado vibratorio de la energía psíquica, a un estado de armonía que condicionará la salud física y mental.

\section{EL TERAPEUTA Y EL PROCESO PSICOTERAPÉUTICO}

El terapeuta es una persona capacitada, entrenada y preparada para poder llevar a cabo el trabajo de psicoterapia, sumado a ello tiene que haber pasado por el proceso psicoterapéutico, en condición de paciente, para poder resolver sus propios conflictos; no cabe duda, que una persona que no haya resuelto sus problemas, pueda resolver el problema de otros. Desde la óptica del psicoanálisis activo, el terapeuta que ha resuelto sus conflictos, fluye en él la energía libremente; durante el proceso psicoterapéutico, ningún problema de su paciente lo perturba; su energía fluye armoniosamente que le permite hacer bien su trabajo. Por eso el contacto terapeuta paciente debe ser, en el plano sutil, lo más íntimo posible, el terapeuta no teme atacar, si es necesario, las defensas del paciente.

Si una persona está en terapia, es porque de alguna manera no ha sentido el placer de la comunicación empática con la madre; lo que el terapeuta debe procurar, es, no sólo sentir el placer de estar en contacto con su paciente, sino también hacer posible que el paciente perciba este placer y lo disfrute dentro del proceso psicoterapéutico. El amor es una energía que irradia, el placer lo atestigua. El terapeuta dispone de buena energía, bajo la forma armoniosa, esta energía es la que tiene el poder de rearmonizar la energía sutil del paciente, y de redirigir el sentido de su flujo.

Antes de ser un fenómeno psicológico, la cura es un evento energético, se dice entonces que el fundamento de la cura es energético, en lo que hace falta alcanzar primero, para rearmonizar la energía que se ha vuelto caótica por los traumatismos pasados.

Cuando el contacto físico empático se impone, hay una conexión directa con esta energía y moviliza un conjunto de emociones variadas, revividas, donde el diálogo paciente- terapeuta se enriquece mucho. Sólo de manera indirecta el contacto verbal, que es indispensable, permite este contacto energético; las 
palabras verdaderas posibilitan la toma de conciencia, el insight, que es un fenómeno energético.

Lo que cura es un campo energético que se percibe como presencia, conciencia, comunicación o amor. El contacto físico empático polariza este campo y dinamiza su efecto. El terapeuta apunta mayormente su presencia sobre este contacto físico, vehículo de este amor que cura. Una energía misteriosa está en juego.

En el paciente la energía es sutil, viva, está constantemente disponible; es la que cumplirá el trabajo de curación. El terapeuta cumplirá la función de quitar los obstáculos a su libre funcionamiento. La energía del terapeuta va a dar a la del paciente el complemento de fuerza que le hace falta para tomar su cauce natural y armonizarse.

Esta energía debe de ser una expresión de amor, pero también de verdad, no es bueno separar el amor a la verdad. Amor, empatía, insight, son tres aspectos de un mismo fenómeno energético, representan el motor de la cura.

Se puede resumir expresando que las funciones del terapeuta en un proceso de psicoanálisis activo son:

Fomenta el contacto físico, aquí el terapeuta conserva al paciente entre sus brazos y lo mantiene para ayudar a vivir una emoción penosa. El con-tacto, es el arte de trabajar con su paciente con mucho tacto, cualidades que tiene que desarrollar todo terapeuta, éste con-tacto se desarrolla en función a la empatía que tiene el terapeuta. El con-tacto se refiere a tener mucho tacto, como también saber tocar al paciente en el momento oportuno, cuando éste lo necesita.

Plantea una comunicación integral, verbal, corporal y afectiva.

Establece una relación empática. La empatía es la que permite al niño llegar a ser consciente de él mismo y construir su identidad.

Presenta una neutralidad que está al servicio del paciente; se entrega, se compromete y al mismo tiempo es espejo, nunca se identifica totalmente con su paciente, esta neutralidad facilita al paciente estar en contacto con el terapeuta. La neutralidad del terapeuta es benévola, es como un espejo que refleja sin deformar las proyecciones del paciente; el terapeuta no juzga ni critica. Esta actitud es importante, porque el terapeuta puede realizar variaciones durante el proceso de acuerdo a su experiencia, teniendo como base esta neutralidad. La neutralidad no está al servicio del miedo, la neutralidad no impide una total libertad, ni todas las actitudes activas.

Tiene la audacia de empujar poco a poco al paciente al transgredir mentalmente todas sus prohibiciones, morir, matar, volverse loco, tener vergüenza, ser castigado si siente placer, etc.

Le incita a vivir sus estados emocionales displacenteros, hasta elaborarlos y reorganizarlos.

El paciente evidencia sus contenidos a través del material, este material es corporal, emocional y verbal; el terapeuta va a recibir, captar y entender a través de la interpretación que el inconsciente del paciente le sugiere y le confía. Lo que el terapeuta le va a decir a su paciente, no es un sencillo eco de las palabras del paciente, sino que el terapeuta también le habla, bajo el entendimiento de la interpretación. Lo que se puede decir que las palabras del terapeuta tienen un valor de iniciación.

La interpretación que practica el terapeuta es la empática, el terapeuta usa la fuerza del verbo o la palabra y el valor de la interpretación justa.

La comprensión, es otro elemento importante en el proceso de la psicoterapia, ésta procede del estado relativamente tardío, donde el niño se reconoció distinto a su madre, y donde su mental adquirió un cierto desarrollo, de la misma manera el paciente tiene que sentir que su terapeuta es muy importante para él, y a la vez son distintos.

En todo proceso psicoterapéutico, se da la regresión, gracias a ella la empatía del terapeuta alcanza al ser en lo más profundo y hace resurgir la energía de curación, en la dirección del momento presente, lo que tiene para restablecer la energía del paciente en su estado natural y armonioso.

En el psicoanálisis activo, el ataque a las defensas es prioritario. Actúa con fuerza y abre las compuertas de la emoción de par en par; hace falta dejarla explayarse, dejar subir los primales, drenar y descargar 
los inmensos flujos de energía que contienen, descender con el paciente hasta el fondo de la regresión y recibir con él todos los dramas, incluso en los rincones más pequeños.

\section{CARACTERÍSTICAS DEL PSICOANÁLISIS ACTIVO: \\ 1.- Es fundamentalmente no directivo. \\ 2.- Está centrado sobre el análisis interpretativo de la transferencia.}

3.- Da importancia al análisis de la interpretación empática, en la regresión.

4.- En la psicoterapia psicoanalítica activa, el contacto es constante y prioritario con el yo del paciente; el empeño y la proximidad intensa del terapeuta permiten una regresión donde las energías primales cortadas y reprimidas se liberan.

5.- El terapeuta deja de ser rigurosamente neutro, contesta a los deseos regresivos que el paciente transfiere sobre él y se compromete totalmente con la contraregresión que le inspira.

Paciente y terapeuta reviven juntos los dramas originales de la infancia del paciente, lo que contribuye aborrarlos.

6.- Es un análisis continuo de todas las defensas y de las actitudes corporales, levanta tarde o temprano el corte.

7.- El secreto del poder del psicoanálisis activo radica en permanecer siempre abierto, hasta el último día.

8.- La empatía es necesaria para comprender al paciente en toda su misteriosa profundidad. La profundidad hasta donde el terapeuta está capacitado para conducir a su paciente, es la misma a la que el terapeuta es llevado por el paciente a descender.

9.- El psicoanálisis activo ofrece un universo total, más natural, un universo no verbal de los intercambios corporales, emocionales y de los primales.

10.- En el psicoanálisis activo, se plantea una comunicación integral, acepta plenamente la regresión y se compromete a fondo.

11.- El terapeuta es capaz de calmar las olas emocionales que emanan del mental del paciente. Por ello no se identifica con su propia conciencia, se sitúa en una posición de testigo y se estabiliza.
12.- El terapeuta no aborda al paciente con ideas preconcebidas u opciones. Es perfectamente neutral, su mente está vacía, sin expectativas, sin deseos.

13.- La herramienta de trabajo del terapeuta es la expresión libre, sin intervención de sus manifestaciones personales.

14.- La primera obligación del terapeuta consiste en dejar una distancia entre su paciente y él. Dentro de esta distancia es donde se efectuará la esencia del trabajo. De este espacio sacará las indicaciones de lo que debe hacer y no hacer, decir y no decir. Es ahí donde encuentra las informaciones necesarias para el buen desarrollo de la sesión.

15.- Desde el principio el terapeuta ama a su paciente (amor terapéutico) espontáneamente y sin esfuerzo.

16.- Antes de comenzar la terapia es necesario asegurarse de que se ha establecido un buen contacto con el yo consciente del paciente, para incitar a este último a asumir la responsabilidad de sí mismo y de su tratamiento, sin proyectarla sobre el terapeuta.

17.- El psicoanálisis activo es un psicoanálisis abierto, salido de sus erranzas pero fiel a su esencia.

18.- El terapeuta activo, cuando se entera de que su paciente transfiere un evento traumático reprimido, dramatiza el evento, lo juega, e induce al paciente a caer en su fuego siempre manteniendo una relación afectuosa con él. Cada sesión es una nueva improvisación.

19.- El terapeuta activo, mantiene un contacto estrecho, en todo momento con el paciente; el contacto psíquico es a menudo reforzado por un contacto físico.

20.- El terapeuta activo, gratifica el esfuerzo, la dedicación, el compromiso del paciente que asume en la terapia; como también gratifica cuando el paciente regresiona al lugar libremente en relación a esos contenidos reprimidos.

\section{REFERENCIAS}

Balint, M. (1934). Análisis del carácter y nuevos comienzos. Londres: Hogarth.

Bleger, J. (1981). Simbiosis y ambigüedad. Buenos Aires: Paidos. 
Blos, P. (1963). Psicoanálisis de la adolescencia. México D.F.: Joaquín Mortiz.

Blum, H. (1976). Acting out. El proceso psicoanalítico y la interpretación. Rev. de Psicoanálisis, 4, 163184.

Boesky, D. (1982). Acting Out. Una Reconsideración del Concepto. Rev. de Psicoanálisis. 63, 39 - 55.

Coderch, J. (1987). Teoría y técnica de la psicoterapia psicoanalítica. Barcelona: Herder.

Ferenczi, S. (1925). El desarrollo del psicoanálisis. Barcelona: Madison.

Freud, S. (1981). Fragmento de análisis de un caso de histeria (Trad. L. López- Ballesteros) Obras Completas (Vol. 1, pp. 933-996) (4ㄹ Ed.). Buenos Aires, Argentina. Biblioteca Nueva. (Trabajo Original publicado en 1905).

Freud, S. (1981). Recuerdo, repetición y elaboración (Trad. L. López- Ballesteros) Obras Completas (Vol. 2, pp. 1683-1688) (4⿳亠口冋 Ed.). Buenos Aires, Argentina: Biblioteca Nueva. (Trabajo Original publicado en 1914).
Freud, S. (1981). Conferencias introductorias al psicoanálisis (Trad. L. López-Ballesteros) Obras Completas (Vol. 3, pp. 2512 - 2518) (4를 Ed.). Buenos Aires, Argentina: Biblioteca Nueva. (Trabajo Original publicado en 1916).

Janov, A. (1970). El grito primal. Buenos Aires: Sudamericana.

Laplanche, J. y Pontalis, J. (1972). Diccionario de psicoanálisis. Barcelona: Labor.

Miller, A. (1983). Por tu propio bien. Barcelona: Tusquets.

Peyrot, S. (1988). Terapia morfoanalítica. Bilbao: DDB.

Reich, W. (1940). Análisis del carácter. Buenos Aires: Paidos.

Rosenfeld, H. (1965). Una investigación en la necesidad del neurótico y el psicótico. Londres: Hogarth.

Sarkissof, J. (1989). Cuerpo y psicoanálisis. Bilbao: DDB. 